\title{
Two issues of the UEFA Euro 2020 qualifying play- offs
}

\section{László Csató}

To cite this article: László Csató (2020): Two issues of the UEFA Euro 2020 qualifying play-offs, International Journal of Sport Policy and Politics, DOI: 10.1080/19406940.2020.1780295

To link to this article: https://doi.org/10.1080/19406940.2020.1780295

册 Published online: 25 Jun 2020.

Submit your article to this journal 준

Q View related articles $\sqsubset$

View Crossmark data 


\section{Two issues of the UEFA Euro 2020 qualifying play-offs}

\section{László Csató}

Research Group of Operations Research and Decision Systems, Laboratory on Engineering and Management Intelligence, Institute for Computer Science and Control (SZTAKI), Budapest, Hungary; Department of Operations Research and Actuarial Sciences, Corvinus University of Budapest (BCE), Budapest, Hungary

\begin{abstract}
The play-offs of the UEFA Euro 2020 qualifying tournament determine the last four participants in the UEFA European Championship 2020. Sixteen teams, which have failed to obtain a slot in the qualifying group stage, will be selected and divided into four paths of four teams each based on the inaugural season 2018-19 of the UEFA Nations League. We provide a critical examination of the relevant UEFA regulation and show that its articles contradict each other and allow for an unfair formation of the playoff paths: it might happen that all conditions cannot be satisfied simultaneously and a group winner might face stronger opponents than a nongroup winner from the same league despite its better performance in the UEFA Nations League. Simple and straightforward solutions for both problems are suggested.
\end{abstract}

\section{ARTICLE HISTORY}

Received 11 July 2019

Accepted 4 June 2020

\section{KEYWORDS}

Consistency; fairness; mechanism design; sports rules; UEFA Euro

Tel brille au second rang, qui s'eclipse au premier. ${ }^{1}$

(Voltaire: La Henriad)

\section{Introduction}

The 2020 UEFA European Football Championship, commonly known as UEFA Euro 2020 or simply Euro 2020, is the official championship of men's national football teams, organised by the Union of European Football Associations (UEFA). ${ }^{2}$ Its qualifying play-offs provide a secondary route for the teams to compete in the final tournament. In contrast to previous editions, the contestants of the play-offs are not determined by the results of the qualifying group stage but based on the inaugural season 2018-19 of the UEFA Nations League (UNL). Therefore, the relevant regulation (UEFA 2018a) has got some attention even in the mainstream media (Guyon 2019b). Since these rules have never been used before but are rather complicated, they may contain more imperfections than usual. The current note will identify two potential problems.

Analysis of sports rules from a theoretical perspective remains an important duty of scientific research since tournament design is a matter of significant financial concern for all stakeholders, and a matter of personal interest in millions of fans (Szymanski 2003). As sports are of great interest to a high percentage of the world's population, they are important topics to be researched into (Wright 2014).

It is well known that sports rules sometimes suffer from unforeseen consequences (Kendall and Lenten 2017). The issue seems to be especially relevant for the UEFA, the governing body of association football in Europe, as has been presented recently. According to Dagaev and Sonin (2018), qualification for the UEFA Europa League was incentive incompatible until the 2015-16 season, namely, a team might 
have benefited from losing instead of winning. This problem occurred in a match of the 2011-12 season of the Eredivisie, the highest echelon of professional football in the Netherlands (Csató 2019b). Because of the same reason, qualification for the UEFA Champions League did not satisfy incentive compatibility in the three seasons between 2016 and 2019 (Csató, 2019a). The qualification for some recent FIFA World Cups and UEFA European Championships (Csató 2018) also allowed for such manipulation but could be eliminated with a marginal amendment of the rules (Csató 2020b). Furthermore, it is even possible that the two teams playing a match are both interested in a draw (Csató 2020c). Guyon (2018) finds several flaws in the design of the UEFA Euro 2016 and suggests two fairer procedures. UEFA has used these results to modify the knockout bracket of the UEFA Euro 2020 in order to reduce its unfairness. Guyon (2019a) presents a new knockout format for the UEFA Euro 2020, which can maximise the number of home games. Finally, Haugen and Krumer (2019) argue that tournament design is an integral part of sports management, and demonstrate a number of shortcomings of the UEFA Euro 2020 qualification, including the violation of a natural fairness criterion (Csató 2020a). In particular, two issues will be discussed: in certain cases, the rules of the UEFA Euro 2020 qualifying play-offs lead to a contradiction and allow for an unfair formation of the play-off paths. Although they may occur with a low probability, UEFA should plan for all possible scenarios without assuming that lower-ranked teams could not perform above their abilities, similarly to any sports governing body.

We think that both potential shortcomings should be addressed not only to ensure the conditions of consistency and fairness but also because they have a political dimension. First, the violation of logical inconsistency can be resolved only by an arbitrary decision, which might have a large reputational cost. Second, any UEFA member association may disapprove if its national team should contest a play-off path regarded unfair. Therefore, besides meeting important theoretical requirements, the implementation of the amendments suggested by us can avoid future criticism from crucial stakeholders, too.

The note is organised as follows. Section 2 shortly outlines the qualification for the UEFA Euro 2020 and presents the rules of the play-offs. Two problems are identified in Section 3. Section 4 proposes solutions to these issues, while Section 5 concludes.

\section{The play-offs of the UEFA Euro 2020 qualifying tournament}

The UEFA Euro 2020 qualifying tournament is a football competition played to select the 24 men's national teams from the 55 UEFA members that will participate in the UEFA European Championship 2020 final tournament.

The qualification is strongly connected to the 2018-19 UEFA Nations League. This competition divides the national teams into four divisions called leagues according to their UEFA coefficients at the end of the 2018 FIFA World Cup qualifiers without the play-offs: the 12 highest-ranked teams form League $A$, the next 12 form League $B$, the next 15 form League $C$, and the remaining 16 form League $D$. The teams of a league play a home-away (double) round-robin tournament in four groups. After ranking the teams in each group, four league rankings are established and aggregated into the overall UEFA Nations League ranking. In particular, the teams of League A occupy positions 1st to 12th based on the ranking in League $A$, the teams of League $B$ occupy positions 13th to 24th based on the ranking in League $B$, the teams of League $C$ occupy positions 25 th to 39th based on the ranking in League $C$, and the teams of League $D$ occupy positions 40 th to 55 th based on the ranking in League $D$. This note will denote all teams by these numbers.

Table 1 provides an overview of the qualification for the UEFA Euro 2020. The whole process is regulated by two official documents (UEFA 2018a, 2018b). It is also discussed in Csató (2020a). The UEFA Euro 2020 qualifying tournament, organised between March 2019 and November 2019, allocates the teams into 10 groups: Groups A-D get one team from the UNL Pot each, and one team from Pots 2-5 each, Group E gets one team from Pots 1-5 each, while Groups F-J get one team from Pots 1-6 each (see Table 1 for the composition of the pots). From all groups, the top two, thus altogether 20 , teams qualify.

The last four participants of the UEFA Euro 2020 are determined by the play-offs of the UEFA Euro 2020 qualifying tournament. Unlike previous editions of the UEFA European Championship, teams 
Table 1. An overview of the qualification for the UEFA Euro 2020.

\begin{tabular}{lccc}
\hline UNL rank & League & $\begin{array}{c}\text { Place in the seeding of the } \\
\text { UEFA Euro 2020 qualifiers }\end{array}$ \\
\hline $1-4(G W)$ & A & UNL Pot & Remark \\
$5-10$ & A & Pot 1 & - \\
$11-12$ & A & Pot 2 & - \\
$13-16(G W)$ & B & Pot 2 & Assured of at least play-offs \\
$17-20$ & B & Pot 2 & - \\
$21-24$ & B & Pot 3 & - \\
$25-28(G W)$ & C & Pot 3 & Assured of at least play-offs \\
$29-30$ & C & Pot 3 & - \\
$31-39$ & C & Pot 4 & - \\
$40(G W)$ & D & Pot 4 & Assured of at least play-offs \\
$41-43(G W)$ & D & Pot 5 & Assured of at least play-offs \\
$44-50$ & D & Pot 5 & - \\
$51-55$ & D & Pot 6 & - \\
\hline
\end{tabular}

GW stands for group winner in the UEFA Nations League

do not advance to the play-offs from the qualifying group stage, but they are selected based on their performance in the 2018-19 UEFA Nations League as follows (UEFA 2018a, Articles 16.01-16.03):

Sixteen teams enter the play-offs, which are played in four separate paths of four teams each, to determine the remaining four teams that qualify for the final tournament.

To determine the 16 teams that enter the play-offs, the following principles apply in the order given:

(a) Four play-off slots are allocated to each league from UEFA Nations League D to UEFA Nations League A, i.e. in reverse alphabetical order.

(b) The UEFA Nations League group winners enter the play-offs unless they have qualified for the final tournament directly from the qualifying group stage.

(c) If a UEFA Nations League group winner has directly qualified for the final tournament, the next best-ranked team in the relevant league ranking which has not directly qualified will enter the play-offs.

(d) If fewer than four teams from one league enter the play-offs, the remaining slots are allocated on the basis of the overall UEFA Nations League rankings to the best-ranked of the teams that have not already qualified for the final tournament, subject to the restriction that group winners cannot be in a play-off path with higher-ranked teams.

The UEFA administration conducts a draw to allocate teams to the different play-offs path, starting with UEFA Nations League $D$, subject to the following conditions:

(a) A group winner cannot form a path with a team from a higher-ranked league in the overall UEFA Nations League rankings.

(b) If four or more teams from a league enter the play-offs, a path with four teams from the league in question must be formed.

(c) Additional conditions may be applied, subject to approval by the UEFA Executive Committee, including seeding principles and the possibility of final tournament host associations having to be drawn into different paths.

For the sake of simplicity, UEFA (2018a, Article 16.02) is called the team selection rule, while UEFA (2018a, Article 16.03) is called the path formation rule in the following.

The four teams of a play-off path play such that the highest-ranked team is matched with the lowest-ranked team and the two middle teams are matched against each other in the semi-finals, hosted by the higher-ranked teams, while the final is contested by the winners of the semi-finals, and is hosted by the winner of a semi-final drawn in advance (UEFA 2018a, Article 17). The winner of this match qualifies for the UEFA Euro 2020.

The rules above are illustrated by the real results.

Example 2.1. Figure 1(a) outlines the situation at the end of the UEFA Euro 2020 qualifying tournament in November 2019. No team qualified from League $D$, hence the four group winners (40-43) form Path D in the play-offs (UEFA 2018a, Article 16.03b). From the four group winners in League C, only team 28 
a The teams that are directly qualified or selected for the play-offs

\begin{tabular}{cccc}
\hline \multicolumn{4}{c}{ League A } \\
A1 & A2 & A3 & A4 \\
\hline$\underline{1}$ & $\frac{2}{6}$ & $\frac{3}{7}$ & $\frac{4}{8}$ \\
$\underline{5}$ & $\underline{6}$ & $\underline{7}$ & $\underline{12}$ \\
$\underline{9}$ & $\underline{10}$ & $\underline{11}$ & \\
\hline \multicolumn{4}{c}{ League $\mathbf{C}$} \\
C1 & C2 & C3 & C4 \\
\hline \multirow{2}{*}{25} & 26 & 27 & $\underline{28}$ \\
29 & 30 & 31 & 32 \\
33 & 34 & 35 & 36 \\
& 37 & 38 & 39 \\
\hline
\end{tabular}

\begin{tabular}{|c|c|c|c|}
\hline \multicolumn{4}{|c|}{ League B } \\
\hline B1 & $\mathrm{B} 2$ & B3 & B4 \\
\hline 13 & 14 & $\underline{15}$ & $\underline{16}$ \\
\hline$\underline{17}$ & $\underline{18}$ & $\underline{19}$ & $\underline{20}$ \\
\hline 21 & $\underline{22}$ & $\overline{23}$ & 24 \\
\hline \multicolumn{4}{|c|}{ League D } \\
\hline D1 & D2 & D3 & $\mathrm{D} 4$ \\
\hline 40 & 41 & 42 & 43 \\
\hline 44 & 45 & 46 & 47 \\
\hline 48 & 49 & 50 & 51 \\
\hline 52 & 53 & 54 & 55 \\
\hline
\end{tabular}

b The path formation

\begin{tabular}{|c|c|}
\hline \multicolumn{2}{|c|}{ Path A } \\
\hline 12 & 32 \\
\hline 29 & 31 \\
\hline
\end{tabular}

\begin{tabular}{ll}
\hline \multicolumn{2}{c}{ Path } \\
\hline 13 & 24 \\
21 & 23 \\
\hline
\end{tabular}

\begin{tabular}{cc}
\hline \multicolumn{2}{c}{ Path } \\
\hline 25 & 30 \\
26 & 27 \\
\hline
\end{tabular}

\begin{tabular}{cc}
\hline \multicolumn{2}{c}{ Path D } \\
\hline 40 & 43 \\
41 & 42 \\
\hline
\end{tabular}

Italic numbers indicate the 16 Nations League group winners

Blue underlined numbers indicate the 20 teams directly qualified

Red bold numbers indicate the 16 teams selected for the play-offs

Figure 1. The result of the qualification for the UEFA Euro 2020.

has obtained a quota, therefore team 29 enters the play-offs (UEFA 2018a, Article 16.02 c). From League $B$, four teams $(13,21,23,24)$, among them a group-winner, have failed to qualify, they form Path $B$ in the play-offs (UEFA 2018a, Article 16.03b). From League A, only team 12 has failed to qualify. According to (UEFA 2018a, Article 16.02d), the remaining three slots in the play-offs are allocated to the highestranked teams that failed to qualify, which are teams 30-32 from League $C$. The team selection rule is detailed in Figure $A 1$ in the Appendix via this example.

Forming Paths $A$ and $C$ has required a draw. UEFA (2018a, Article 16.03a) provides that the three group winners in League $C(25-27)$ are in Path $C$, and team 30 has been drawn there. Consequently, Path $A$ is formed by teams $12,29,31$, and 32 as Figure 1 (b) shows.

In each path, the semi-finals are hosted by the two higher-ranked teams, for example, team 29 plays against team 31 at home. According to the random draw, the winner of this semi-final hosts the final of Path A.

The path formation rule is detailed in Figure $\mathrm{A} 2$ in the Appendix via this example. 


\section{The problems of the regulation}

We formulate here two reasonable conditions and show that they are not guaranteed by the rules of the UEFA Euro 2020 qualifying play-offs.

\subsection{Consistency}

The first requirement deals with the logical connections between the criteria of team selection.

Definition 3.1. Consistency: A team selection rule is called consistent if its principles do not contradict each other. Otherwise, it is said to be inconsistent.

Inconsistency means a problem because a possible scenario not addressed by the rules necessitates an arbitrary decision, which can lead to long controversies such as in the case of the qualification of the titleholder Liverpool F.C. for the 2005-06 UEFA Champions League.

Proposition 3.2. The team selection rule of UEFA (2018a, Article 16.02) is inconsistent.

Proof. It is sufficient to provide an example where the principles are contradictory.

Figure 2(a) outlines such a scenario. Teams 40-42 are group winners in League $D$, therefore they enter the play-offs. However, since all other teams in League $D$ have already been qualified, the three group winners in League $D$ should be in a play-off path with a higher-ranked team, which is not allowed by UEFA (2018a, Article 16.02d).

Independently of us, this issue has been identified on an online football forum. ${ }^{3}$

\subsection{Fairness}

The second condition concerns the strength of play-off paths. First, note that the rules differentiate between the leagues and prefer group winners, therefore the teams can be divided into a hierarchy of eight levels: teams 1-4 (group winners in League A); teams 5-12 (non-group winners in League A); teams 13-16 (group winners in League B); teams 17-24 (non-group winners in League B); teams 25-28 (group winners in League C); teams 29-39 (non-group winners in League C); teams 40-43 (group winners in League D); and teams 44-55 (non-group winners in League D).

A team that has a higher place in this hierarchy is called stronger. The implied binary relation is denoted by $\geq: I \geq j$ if team $i$ is at least as strong as team $j, i \sim j$ if team $i$ is at the same level of the hierarchy as team $j$, and $i \succ j$ if team $i$ is stronger than team $j$.

The play-off paths are represented by the four teams that form them.

Definition 3.3. Difficulty of play-off paths: Consider two teams $i, j$ participating in different play-off paths $P_{i}=\left\{i, i_{1}, i_{2}, i_{3}\right\}$ and $P_{j}=\left\{j, j_{1}, j_{2}, j_{3}\right\}$, where $i_{1}<i_{2}<i_{3}$ and $j_{1}<j_{2}<j_{3}$. The play-off path $P_{i}$ of team $i$ is more difficult than the play-off path $P_{j}$ of team $j$ if one of the following holds:

(1) $i_{1} \geq j_{1}, i_{2} \geq j_{2}$, and $i_{3} \geq j_{3}$, furthermore, $i_{m} \succ j_{m}$ for at least one $1 \leq m \leq 3$; or

(2) $i_{1} \succ j_{1}$, and it is not true that team $i$ plays at home but team $j$ plays away in the semi-finals of the corresponding play-off paths.

Condition i) of Definition 3.3 is straightforward as it is more difficult to qualify against a set of better teams. Condition ii) can be necessary because the play-off path $P_{i}$ is certainly more difficult than the playoff path $P_{j}$ if a stronger team always defeats a weaker one, and the strongest opponent in a play-off path is 


\section{a A feasible scenario}

\begin{tabular}{|c|c|c|c|}
\hline \multicolumn{4}{|c|}{ League A } \\
\hline A1 & $\mathrm{A} 2$ & A3 & A4 \\
\hline 1 & $\underline{2}$ & $\underline{3}$ & 4 \\
\hline$\overline{5}$ & $\overline{6}$ & $\overline{7}$ & $\frac{1}{8}$ \\
\hline 9 & 10 & 11 & 12 \\
\hline \multicolumn{4}{|c|}{ League C } \\
\hline $\mathrm{C} 1$ & $\mathrm{C} 2$ & $\mathrm{C} 3$ & $\mathrm{C} 4$ \\
\hline 25 & 26 & 27 & 28 \\
\hline 29 & 30 & 31 & 32 \\
\hline \multirow[t]{2}{*}{33} & 34 & 35 & 36 \\
\hline & 37 & 38 & 39 \\
\hline
\end{tabular}

\begin{tabular}{cccc}
\hline \multicolumn{4}{c}{ League B } \\
B1 & B2 & B3 & B4 \\
\hline 13 & 14 & 15 & 16 \\
$\frac{17}{21}$ & $\underline{18}$ & $\underline{19}$ & 20 \\
22 & 23 & 24 \\
\hline & \multicolumn{4}{c}{ League D } \\
\hline D1 & D2 & D3 & D4 \\
\hline 40 & 41 & 42 & $\underline{43}$ \\
$\frac{44}{48}$ & $\underline{45}$ & $\underline{46}$ & $\underline{47}$ \\
$\underline{52}$ & $\underline{53}$ & $\underline{54}$ & $\underline{51}$ \\
\hline
\end{tabular}

b The suggested path formation

\begin{tabular}{|c|c|}
\hline \multicolumn{2}{|c|}{ Path A } \\
\hline 5 & 8 \\
\hline 6 & 7 \\
\hline
\end{tabular}

\begin{tabular}{|c|c|}
\hline \multicolumn{2}{|c|}{ Path B } \\
\hline 13 & 16 \\
\hline 14 & 15 \\
\hline
\end{tabular}

\begin{tabular}{cc}
\hline \multicolumn{2}{c}{ Path } \\
$\mathbf{C}$ \\
\hline 25 & 28 \\
26 & 27 \\
\hline
\end{tabular}

\begin{tabular}{ll}
\hline \multicolumn{2}{c}{ Path } \\
\hline
\end{tabular}

Italic numbers indicate the 16 Nations League group winners

Blue underlined numbers indicate the 20 teams directly qualified

Red bold numbers indicate the 16 teams selected for the play-offs

Figure 2. The team selection rule of the play-offs can be inconsistent.

the likely contestant in the final. The host country is taken into account because home advantage is a wellattested phenomenon in international football (Baker and McHale 2018).

This order between the play-off paths is not complete: it might happen that the play-off path $P_{i}$ of team $i$ is not more difficult than the play-off path $P_{j}$ of team $j$, and vice versa.

According to the underlying idea of UEFA (2018a, Article 16), the group winners of the UEFA Nations League are preferred in the play-offs as they cannot form a path with a higher-ranked (stronger) team. However, this requirement still does not guarantee intra league monotonicity, which motivates the following fairness condition.

Definition 3.4. A path formation rule is unfair if a group winner may be in a more difficult play-off path than a non-group winner of the same league.

Although the qualification is intentionally designed in such a way that teams from League $D$ can obtain a place in the UEFA Euro 2020 at the expense of higher-ranked teams from Leagues A, B or C, we think that (possibly) punishing a group winner for its better performance in the UEFA Nations League compared to a team from the same league is hard to explain. 
Since condition ii) of Definition 3.3 is a less obvious requirement compared to condition i), it may be even left out from the interpretation of unfairness. Furthermore, analogously to the suggestion of Guyon (2019a), it is possible to allow for the group winner to decide whether it has a more difficult play-off path, and to choose the one which is judged more favourable by the team.

Proposition 3.5. The path formation rule of UEFA (2018a, Article 16.03) is unfair.

Proof. It is sufficient to provide an example where the principles do not guarantee fairness.

Figure 3(a) outlines such a scenario that has been 'officially presented' in UEFA (2017, European qualifiers play-offs scenario 1), together with a possible path formation shown in Figure $3(\mathrm{~b})$. The play-off Path B of team 13 is more difficult than the play-off Path A of team 17 due to condition i) of Definition 3.3 as $20 \sim 23,21 \succ 29$, and $24 \succ 30$, furthermore, both teams play the semi-final at home. However, team 13 is a group winner in League $B$ and team 17 is non-group winner in League $B$; therefore, this path formation, allowed by the regulation, is unfair: qualification through the play-offs will be more difficult for team 13 than for team 17 .

Fairness can be achieved by exchanging teams 13 and 17 as presented in Figure 3(c). This unfairness is not realised in UEFA (2017, European qualifiers play-offs scenario 1). The following example illustrates why condition ii) of Definition 3.3 can be necessary.

Example 3.6. Consider the case shown in Figure 4(a) and the path formation shown in Figure 4(b). Condition i) of Definition 3.3 is not violated because the group winners in League $B$ (teams 13, 14) face one group winner from League $B$ and two teams from League $C$ (teams 29, 30) in Path $A$, while a non-group winner in League $B$ (teams $19,21,23,24)$ faces three teams from League $B$ in Path B.

However, there are two group winners from League B in Path A (both playing at home in the semifinal) but none in Path $B$, which seems to be unfair because they can play against each other in the final of Path A. Condition ii) of Definition 3.3 holds for Path A of team 14 and Path B of team 19 since $13 \succ 21$. Hence, Figure 4 (b) corresponds to an unfair path formation.

Fairness can be achieved by exchanging teams 14 and 19 as presented in Figure 4(c).

\section{The proposed solutions}

This section provides our amendments to solve the issues discussed in Section 3.

\subsection{Guaranteeing consistency}

The inconsistency of the team selection rule can be handled straightforwardly by supplementing UEFA (2018a, Article 16.02d) with a specific clause:

'If fewer than four teams from one league enter the play-offs, the remaining slots are allocated on the basis of the overall UEFA Nations League rankings to the best-ranked of the teams that have not already qualified for the final tournament, subject to the restriction that group winners cannot be in a play-off path with higher-ranked teams, or, if this is not possible, they form a play-off path with the lowestranked teams that have not already qualified'.

Figure 2(b) shows the result of this proposal: since there are only three teams from League $D$ that failed to qualify directly, and there is at least one group winner among them, the remaining slot in Path $\mathrm{D}$ is allocated to team 39 , the lowest-ranked team that has not already qualified. 
a A possible scenario (UEFA, 2017, European qualifiers play-offs scenario 1)

\begin{tabular}{cccc}
\hline \multicolumn{4}{l}{ League A } \\
A1 & A2 & A3 & A4 \\
\hline$\underline{1}$ & $\underline{2}$ & $\underline{3}$ & $\frac{4}{8}$ \\
$\underline{5}$ & $\underline{6}$ & $\underline{7}$ & $\underline{12}$ \\
$\underline{9}$ & $\underline{10}$ & $\underline{11}$ & $\underline{12}$ \\
\hline \multicolumn{5}{c}{ League $\mathbf{C}$} \\
C1 & C2 & C3 & C4 \\
\hline \multirow{2}{*}{25} & $\underline{26}$ & 27 & 28 \\
29 & 30 & $\underline{31}$ & 32 \\
33 & 34 & 35 & 36 \\
& 37 & 38 & 39 \\
\hline
\end{tabular}

\begin{tabular}{cccc}
\hline \multicolumn{4}{c}{ League B } \\
B1 & B2 & B3 & B4 \\
\hline 13 & $\frac{14}{18}$ & $\frac{15}{19}$ & $\frac{16}{20}$ \\
17 & $\frac{16}{22}$ & 23 & 24 \\
21 & $\underline{4}$ & \\
\hline \multicolumn{4}{l}{ League D } \\
D1 & D2 & D3 & D4 \\
\hline \multirow{4}{*}{40} & 41 & 42 & 43 \\
44 & 45 & 46 & 47 \\
48 & 49 & 50 & 51 \\
52 & 53 & 54 & 55 \\
\hline
\end{tabular}

b An unfair path formation (UEFA, 2017, European qualifiers play-offs scenario 1)

\begin{tabular}{|c|c|}
\hline \multicolumn{2}{|c|}{ Path A } \\
\hline 17 & 30 \\
\hline 23 & 29 \\
\hline
\end{tabular}

\begin{tabular}{|c|c|}
\hline & $\mathbf{A}$ \\
\hline 13 & 30 \\
\hline 23 & 29 \\
\hline
\end{tabular}

\begin{tabular}{ll}
\hline \multicolumn{2}{c}{ Path } \\
B \\
\hline 13 & 24 \\
20 & 21 \\
\hline
\end{tabular}

c A fair path formation

\begin{tabular}{cc}
\hline \multicolumn{2}{c}{ Path $\mathbf{C}$} \\
\hline 25 & 32 \\
27 & 28 \\
\hline
\end{tabular}

\begin{tabular}{ll}
\hline \multicolumn{2}{c}{ Path D } \\
\hline 40 & 43 \\
41 & 42 \\
\hline
\end{tabular}

Italic numbers indicate the 16 Nations League group winners

Blue underlined numbers indicate the 20 teams directly qualified

Red bold numbers indicate the 16 teams selected for the play-offs

Figure 3. The path formation rule of the play-offs may violate the first fairness condition.

\subsection{Guaranteeing fairness}

In order to avoid a possibly unfair path formation, UEFA (2018a, Article 16.03a) is worth supplementing with a further condition:

'A group winner cannot form a path with a team from a higher-ranked league in the overall UEFA Nations League rankings (or, if this is not possible, they form a play-off path with the lowestranked teams that have not already qualified), and cannot be in a more difficult play-off path than a non-group winner of the same league.' 
a A feasible scenario

\begin{tabular}{|c|c|c|c|}
\hline \multicolumn{4}{|c|}{ League A } \\
\hline A1 & $\mathrm{A} 2$ & A3 & $\mathrm{A} 4$ \\
\hline$\underline{1}$ & $\underline{2}$ & $\underline{3}$ & 4 \\
\hline$\underline{5}$ & $\underline{6}$ & $\underline{7}$ & $\underline{\overline{8}}$ \\
\hline$\underline{\overline{9}}$ & $\underline{10}$ & $\underline{11}$ & $\underline{12}$ \\
\hline \multicolumn{4}{|c|}{ League $\mathrm{C}$} \\
\hline $\mathrm{C} 1$ & $\mathrm{C} 2$ & C3 & $\mathrm{C} 4$ \\
\hline 25 & $\underline{26}$ & 27 & 28 \\
\hline 29 & 30 & $\underline{31}$ & 32 \\
\hline \multirow[t]{2}{*}{33} & 34 & 35 & 36 \\
\hline & 37 & 38 & 39 \\
\hline
\end{tabular}

\begin{tabular}{|c|c|c|c|}
\hline \multicolumn{4}{|c|}{ League B } \\
\hline B1 & B2 & B3 & B4 \\
\hline 13 & 14 & $\underline{15}$ & 16 \\
\hline$\underline{17}$ & $\underline{18}$ & 19 & $\underline{20}$ \\
\hline 21 & $\underline{22}$ & 23 & 24 \\
\hline \multicolumn{4}{|c|}{ League D } \\
\hline D1 & D2 & D3 & D4 \\
\hline 40 & 41 & 42 & 43 \\
\hline 44 & 45 & 46 & 47 \\
\hline 48 & 49 & 50 & 51 \\
\hline 52 & 53 & 54 & 55 \\
\hline
\end{tabular}

b An unfair path formation allowed by the rules

\begin{tabular}{|c|c|}
\hline \multicolumn{2}{|c|}{ Path A } \\
\hline 13 & 30 \\
\hline 14 & 29 \\
\hline
\end{tabular}

\begin{tabular}{ll}
\hline \multicolumn{2}{c}{ Path } \\
\hline 19 & 24 \\
21 & 23 \\
\hline
\end{tabular}

\begin{tabular}{cc}
\hline \multicolumn{2}{c}{ Path } \\
$\mathbf{C}$ \\
\hline 25 & 32 \\
27 & 28 \\
\hline
\end{tabular}

\begin{tabular}{ll}
\hline \multicolumn{2}{c}{ Path } \\
\hline
\end{tabular}

c A fair path formation

\begin{tabular}{|c|c|}
\hline \multicolumn{2}{|c|}{ Path A } \\
\hline 13 & 30 \\
\hline 19 & 29 \\
\hline
\end{tabular}

\begin{tabular}{lr}
\hline \multicolumn{2}{c}{ Path B } \\
\hline 14 & 24 \\
21 & 23 \\
\hline
\end{tabular}

\begin{tabular}{cc}
\hline \multicolumn{2}{c}{ Path $\mathbf{C}$} \\
\hline 25 & 32 \\
27 & 28 \\
\hline
\end{tabular}

\begin{tabular}{cc}
\hline \multicolumn{2}{c}{ Path D } \\
\hline 40 & 43 \\
41 & 42 \\
\hline
\end{tabular}

Italic numbers indicate the 16 Nations League group winners

Blue underlined numbers indicate the 20 teams directly qualified

Red bold numbers indicate the 16 teams selected for the play-offs

Figure 4. The path formation rule of the play-offs may violate the second fairness condition.

The rules should also explain the comparison of play-off paths with respect to their difficulty, analogously to Definition 3.3.

It remains a question how fairness can be achieved. We propose to follow the solution used in the proof of Proposition 3.5 and in Example 3.6: if a group winner is in a more difficult play-off path than a non-group winner of the same league, then simply exchange the two teams. Note that this does not violate any principle of the regulation. If there exists more than one appropriate exchange (for instance, both of the teams 13 and 14 have a more difficult play-off path than team 19), it is worth choosing randomly between them. 
According to the following statement, the application of this procedure will provide fairness.

Proposition 4.1. There always exists a fair path formation that can be reached by exchanging group winners with non-group winners of the same league if the former are in a more difficult play-off path than the latter.

Proof. Focus on the sets of opponents $O_{k}=\left\{k_{1}, k_{2}, k_{3}\right\}$ of every team $k$ in League $X$. It can be assumed without loss of generality that $k_{1}<k_{2}<k_{3}$. Define a partial order between the sets $O_{k}=$ $\left\{k_{1}, k_{2}, k_{3}\right\}$ and $O_{\ell}=\left\{\ell_{1}, \ell_{2}, \ell_{3}\right\}$ with respect to their strength on the basis of Definition 3.3, that is, $O_{k}>O_{\text {l }}$ if either $K_{1}>I_{1}, k_{1} \geq I_{1}, k_{2} \geq I_{2}$, and $k_{3} \geq I_{3}$, furthermore, $k_{m} \geq I_{m}$ for at least one $1 \leq m \leq 3$ it is not true that team $k$ plays away but team $\ell$ plays at home in the semi-final. Otherwise, $O_{k} \sim O_{\ell}$.

If the set of teams that are group winners in League $X$ and participate in the play-offs is empty, then fairness holds for this particular league. Otherwise, take team $i$ that is a group winner in League $X$ and $O_{i}>O_{k}$ for all team $k$ that is a group winner in League $X$.

If the set of teams that are non-group winners in League $X$ and participate in the play-offs is empty, then fairness holds for this particular league. Otherwise, take team $j$ that is a non-group

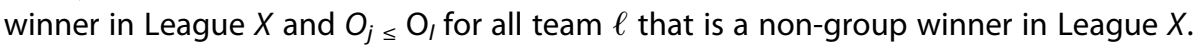

If $O_{i} \leq \mathrm{O}_{j}$, then no group winner in League $X$ has a more difficult play-off path than any non-group winner in League $X$; therefore, fairness holds for this particular league.

If $O_{i} \succ O_{j}$, exchange teams $i$ and $j$ in their paths $P_{i}$ and $P_{j}$. The new paths are $P_{i}^{*}=O_{i} \cup\{j\}$ and $P_{j}^{*}=O_{j} \cup\{i\}$ with the following implications for the new sets of opponents $O_{k}^{*}$ :

- $O_{i}^{*}=O_{j}$ for the particular team $i$ in path $P_{i}$ (and $P_{j}^{*}$ ) that is a group winner in League $X$;

- $O_{k}^{*}=\left(O_{k} \cup\{j\} \backslash\{i\}\right) \prec O_{k}$ for any team $k$ in path $P_{i}$ (and $P_{i}^{*}$ ) that is a group winner in League $X$;

- $O_{\ell}^{*}=\left(O_{\ell} \cup\{j\} \backslash\{i\}\right) \sim O_{i}=O_{j}^{*}$ for any team $\ell$ in path $P_{i}$ (and $P_{i}^{*}$ ) that is a non-group winner in League $X$;

- $O_{j}^{*}=O_{i}$ for the particular team $j$ in path $P_{j}$ (and $P_{i}^{*}$ ) that is a non-group winner in League $X$;

- $O_{k}^{*}=\left(O_{k} \cup\{i\} \backslash\{j\}\right) \sim O_{j}=O_{i}^{*}$ for any team $k$ in path $P_{j}$ (and $P_{j}^{*}$ ) that is a group winner in League $X$;

- $O_{\ell}^{*}=\left(O_{\ell} \cup\{i\} \backslash\{j\}\right) \succ O_{\ell}$ for any team $\ell$ in path $P_{i}$ (and $\left.P_{i}^{*}\right)$ that is a non-group winner in League $X$.

Consequently, $O_{j}$, one of the lowest-ranked sets of opponents for non-group winners in League $X$ becomes stronger, while $O_{i}$, one of the highest-ranked sets of opponents for group winners in League $X$ becomes weaker; therefore, changing teams $i$ and $j$ is a step towards achieving a fair path formation.

Since the number of teams and leagues is finite, this procedure is guaranteed to finish after a finite number of steps; thus, a fair path formation should exist.

An unfair path formation can usually be corrected with only one exchange of teams as we have seen in the proof of Proposition 3.5 and in Example 3.6. Two steps are necessary, for instance, if Path $B$ is composed of teams 13, 14, 15, and 16 (four group winners from League B) and Path A is composed of teams 17, 18, 19 and 29 (three non-group winners from League $B$ and one from League C). We conjecture that two steps are always sufficient but its verification seems to be even more cumbersome than the proof of Proposition 4.1.

It is worth noting here that UEFA does not provide any algorithm for team selection and path formation rules which guarantees that the conditions of UEFA (2018a, Articles 16.01-16.03) hold (UEFA (2017) presents only three possible scenarios). Nonetheless, it would not be a futile exercise because the detailed description of an algorithm would help to identify some problems such as inconsistency. 


\section{Conclusions}

This critical note has investigated the regulation of the UEFA Euro 2020 qualifying play-offs. We have identified two theoretical shortcomings of it, that is, logical inconsistency between the principles and the possible unfair formation of the play-off paths. They, together with the opportunity for strategic manipulation in the UEFA Nations League (Csató 2020a), warn to the dangers inherent in reforming tournament designs with similarly complicated rules.

While the presented issues may occur with a low probability, the integrity of sports rules requires all possible scenarios to be considered in a fair way. Hopefully, our reasoning will convince UEFA that the proposed amendments - which does not increase the complexity of the rules - are worth implementing.

\section{Notes}

1. 'He shines in the second rank, who is eclipsed in the first.' Source: https://quotes.yourdictionary.com/author/quote/ 539979. Downloaded 26 March 2019.

2. On 17 March 2020, UEFA has announced that the tournament would be delayed by a year due to the coronavirus pandemic. The play-off matches have been postponed until further notice on 1 April 2020. Nonetheless, we will refer to this event as UEFA Euro 2020.

3. See the post of the user 'Forza AZ' on 10 October 2017, 23:41 at https://kassiesa.net/uefa/forum2/viewtopic.php? $f=5 t=3463$ start $=150$. We are grateful to an anonymous referee for the remark.

\section{Acknowledgments}

Four anonymous reviewers, Dezsö Bednay, and Tamás Halm have provided valuable comments and suggestions on earlier drafts. We are indebted to the https://en.wikipedia.org/wiki/Wikipedia_communityWikipedia community for contributing to our research by collecting and structuring useful information on the sports tournament discussed.

\section{Disclosure statement}

No potential conflict of interest was reported by the author.

\section{Funding}

The research was supported by the MTA Premium Postdoctoral Research Program grant PPD2019-9/2019.

\section{References}

Baker, R.D. and McHale, I.G., 2018. Time-varying ratings for international football teams. European Journal of Operational Research, 267 (2), 659-666. doi:10.1016/j.ejor.2017.11.042.

Csató, L., 2018. Was Zidane honest or well-informed? How UEFA barely avoided a serious scandal. Economics Bulletin, 38 (1), 152-158.

Csató, L., 2019a. UEFA Champions League entry has not satisfied strategyproofness in three seasons. Journal of Sports Economics, 20 (7), 975-981. doi:10.1177/1527002519833091.

Csató, L., 2019b. When UEFA rules had inspired unfair behaviour on the field. Manuscript. arXiv: 1806.03978.

Csató, L., 2020a. Fair tournament design: a flaw of the UEFA Euro 2020 qualification. Manuscript. arXiv: 1905.03325.

Csató, L., 2020b. The incentive (in)compatibility of group-based qualification systems. International Journal of General Systems, 49 (4), 374-399. doi:10.1080/03081079.2020.1748618.

Csató, L., 2020c. When neither team wants to win: a flaw of recent UEFA qualification rules. International Journal of Sports Science \& Coaching, 174795412092100. in press. doi:10.1177/1747954120921001.

Dagaev, D. and Sonin, K., 2018. Winning by losing: incentive incompatibility in multiple qualifiers. Journal of Sports Economics, 19 (8), 1122-1146. doi:10.1177/1527002517704022.

Guyon, J., 2018. What a fairer 24 team UEFA Euro could look like. Journal of Sports Analytics, 4 (4), 297-317. doi:10.3233/ JSA-180219. 
Guyon, J., 2019a. "Choose your opponent": A new knockout format for sports tournaments. Application to the Round of 16 of the UEFA Champions League and to maximizing the number of home games during the UEFA Euro 2020. Manuscript. doi:10.2139/ssrn.3488832.

Guyon, J., 2019b. Euro 2020: les Bleus condamnés à défier un gros d'Europe dés la phase de poules (Euro 2020: the Blues condemned to challenge a big one from Europe in the group stage (Euro 2020: the Blues condemned to challenge a big one from Europe in the group stage, in French). Le Monde, 20 November. Available from: https://www.lemonde. $\mathrm{fr} /$ sport/article/2019/11/20/euro-2020-les-bleus-condamnes-a-defier-un-gros-d-europe_6019907_3242.html

Haugen, K.K. and Krumer, A., 2019. On importance of tournament design in sports management: Evidence from the UEFA Euro 2020 qualification. Manuscript. Available from: https://www.researchgate.net/publication/337771711.

Kendall, G. and Lenten, L.J.A., 2017. When sports rules go awry. European Journal of Operational Research, 257 (2), 377-394. doi:10.1016/j.ejor.2016.06.050.

Szymanski, S., 2003. The economic design of sporting contests. Journal of Economic Literature, 41 (4), $1137-1187$. doi:10.1257/002205103771800004.

UEFA, 2017. UEFA Nations League Media Briefing. Nyon, 20 September. Available from: https://web.archive.org/web/ 20180905214902/http://files.footballseeding.com/200002130-9283c937e4/UNL\%20media\%20briefing\%20PDF\% 20version.pdf.

UEFA, 2018a. Regulations of the UEFA European Football Championship 2018-20. Available from: https://www.uefa.com/ MultimediaFiles/Download/Regulations/uefaorg/Regulations/02/54/36/05/2543605_DOWNLOAD.pdf.

UEFA, 2018b. Regulations of the UEFA Nations League 2018-19. Available from: https://www.uefa.com/MultimediaFiles/ Download/Regulations/uefaorg/Regulations/02/50/54/37/2505437_DOWNLOAD.pdf.

Wright, M., 2014. OR analysis of sporting rules - A survey. European Journal of Operational Research, 232 (1), 1-8. doi:10.1016/j.ejor.2013.03.043. 


\section{Appendix}

a The (real-world) scenario of Example 2.1: directly qualified teams

League A
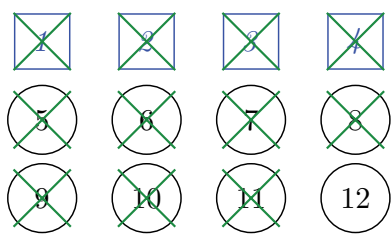

League $\mathrm{C}$
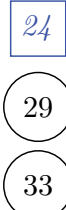

33
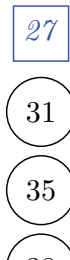
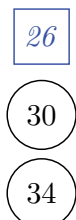

37
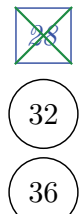

39
League B
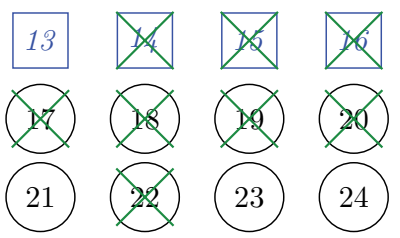

League D
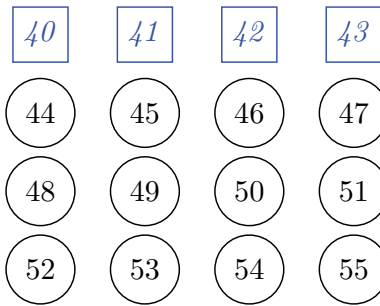

50

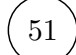

54

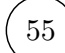

Blue rectangles with italic numbers indicate group winners

Crossed teams indicate directly qualified teams which are unavailable for the play-offs

b The (real-world) scenario of Example 2.1: team selection for the play-offs

Group winners qualify for the play-offs unless they have already qualified

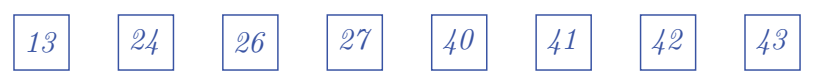

Group winners already qualified are replaced by the next best-ranked team in the same league which has not directly qualified

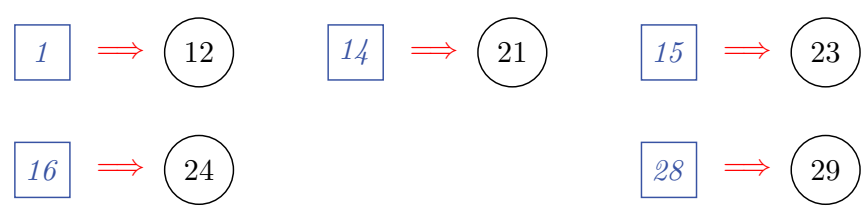

If there is not enough teams in the league, group winners are replaced by the best team in the overall ranking which has not directly qualified BUT: group winners cannot play with teams from a higher league

$$
2 \Longrightarrow 30 \quad 3 \quad 3132
$$

Figure A1. The result of the qualification for the UEFA Euro 2020: team selection. 
a The (real-world) scenario of Example 2.1: teams in the play-offs Blue rectangles with italic numbers indicate group winners

\section{League A}

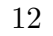

League $\mathrm{C}$

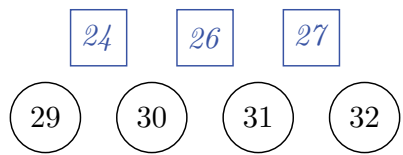

League B

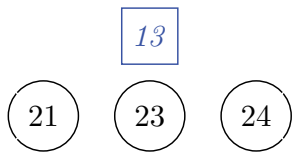

League D

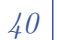

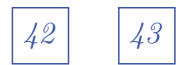

b The (real-world) scenario of Example 2.1: path formation for the play-offs

If four or more teams from a league enter the play-offs, a path with four teams from the league in question must be formed

BUT: a group winner cannot form a path with a team from a higher-ranked league

\begin{tabular}{|c|c|c|c|c|c|}
\hline \multirow[t]{3}{*}{ Path D } & Semifinal 1 (SF1) & 40 & against & 43 & \\
\hline & Semifinal 2 (SF2) & 41 & against & 42 & \\
\hline & Final (host drawn) & $\mathcal{W} / \mathbf{S F} 1$ & against & $\mathcal{W}$ & $\Longrightarrow \mathcal{W}$ \\
\hline \multirow[t]{3}{*}{ Path C } & Semifinal 1 (SF1) & 25 & against & & $\begin{array}{l}\text { drawn } \\
\text { randomly }\end{array}$ \\
\hline & Semifinal 2 (SF2) & 26 & against & 27 & \\
\hline & Final (host drawn) & $\mathcal{W} /$ SF2 & against & $\mathcal{W}$ & $\Longrightarrow \mathcal{W}$ \\
\hline \multirow[t]{3}{*}{ Path B } & Semifinal 1 (SF1) & 13 & against & & \\
\hline & Semifinal 2 (SF2) & & against & & \\
\hline & Final (host drawn) & $\mathcal{W} /$ SF 1 & against & $\mathcal{W} / \mathrm{S}$ & $\Longrightarrow \mathcal{W}$ \\
\hline \multirow[t]{3}{*}{ Path A } & Semifinal 1 (SF1) & & against & & \\
\hline & Semifinal 2 (SF2) & & against & & \\
\hline & Final (host drawn) & $\mathcal{W} / \mathrm{SF} 2$ & against & $\mathcal{W} /$ & $\Longrightarrow \mathcal{W}$ \\
\hline
\end{tabular}

Figure A2. The result of the qualification for the UEFA Euro 2020: path formation. 